\title{
An industry update in therapeutics: what is the latest news?
}

\author{
Catarina P Reis*,1,2 iD \\ ${ }^{1}$ iMED.ULisboa, Research Institute for Medicines, Department of Pharmaceutical Technology (DFGTF), Faculty of Pharmacy, \\ Universidade de Lisboa, Av. Prof. Gama Pinto, 1649-003 Lisboa, Portugal \\ ${ }^{2}$ IBEB, Biophysics \& Biomedical Engineering Institute, Faculty of Sciences, Universidade de Lisboa, Campo Grande, 1749-016, \\ Lisboa, Portugal \\ *Author for correspondence: Tel.: + 351217946429 ext. 14244; Fax: +351 217946 470; catarinareis@ff.ulisboa.pt
}

This industry update covers March of 2019 and describes the most representative examples of research mainly focused on cancer, diabetes, cholesterol-lowering drugs, Parkinson's disease, ischemia and regeneration of heart muscle. A new method for designing encapsulation process and a new in vitro model of oral cavity were also described. In this update, there was a clear positive approval tendency for several companies. Those companies offered innovative therapeutic candidates or in some cases extension of indications. Some patents related to therapeutic fields like lung cancer, diabetes, scleroderma, multiple sclerosis and hypogonadism were also published during this month. Information was sourced from websites, pharma press releases, regulatory and patent agencies, and available scientific literature.

First draft submitted: 8 April 2019; Accepted for publication: 11 June 2019; Published online: 24 June 2019

Keywords: cancer $\bullet$ diabetes $\bullet$ innovation $\bullet$ ischemia $\bullet$ multiple sclerosis $\bullet$ new approvals $\bullet$ Parkinson's disease $\bullet$ patents $\bullet$ research

\section{Research \& development \\ Cell's fingerprint in cancer}

On 11 March, a new strategy to computationally analyze the expression of marker genes as a 'fingerprint' of cancer cells was published. The centrosome is the major microtubule-organizing center of animal cells. The centrosome is essential for several fundamental cellular processes, including cell polarity, signalling, division and migration. It is also implicated in the maintenance of genome stability [1]. A research team used a transcriptomic signature (CA2O) to characterize the landscape of CA-associated gene expression in 9721 tumors. These data were based on The Cancer Genome Atlas. Considering that $C A 20$ is upregulated in cancer, these researchers proposed novel molecular players associated with $\mathrm{CA}$ in cancer. They showed that $C A 20$ upregulation is positively associated with genomic instability, alteration of specific chromosomal arms and $\mathrm{C}>\mathrm{T}$ mutations.

\section{Precision cancer drugs called poly(ADP-ribose) polymerase inhibitors}

Published data showed that $30-50 \%$ of patients with non-small-cell lung cancer show a deficiency in the ERCC1 DNA repair system. Poly(ADP-ribose) polymerase (PARP) is a nuclear enzyme, which is recruited to repair DNA damage. Inhibitors of PARP (PARPi) selectively target cancer cells with DNA repair deficiencies.

A recent study explained how PARPi works [2]. Mechanistically, PARPi generated cytoplasmic chromatin fragments with characteristics of micronuclei. Besides other cell events and reactions, PARPi also potentiated IFN$\gamma$-induced PD-L1 expression. Clinical trials have already started to explore this question, aiming new alternative therapies (combination therapies) for cancer treatment.

\section{A new oral cholesterol-lowering drug}

Bempedoic acid is a small molecule that has been shown to lower the low-density lipoprotein cholesterol levels. The mechanism of action involves the inhibition of ATP citrate lyase. ATP citrate lyase is a key enzyme in the cholesterol biosynthesis pathway. It acts upstream of 3-hydroxy-3-methylglutaryl coenzyme A reductase. 
On 13 March, a randomized, 52-week controlled trial involving 2230 patients with atherosclerotic cardiovascular disease, heterozygous familial hypercholesterolemia, or both was published [3]. In this study, patients had different cholesterol-lowering treatments; it also included a small proportion of patients with familial hypercholesterolemia. After 3 months of treatment, the bempedoic acid reduced low-density lipoprotein cholesterol levels (by an average of $18.1 \%$ from the baseline), confirming that the treatment was effective. This study demonstrated that the treatment was well tolerated by patients.

\section{New insights in Parkinson's disease}

Parkinson's disease is the second most common neurodegenerative disorder. This disease is characterized by loss of dopamine-producing neurons in the substantia nigra pars compacta and formation of intracellular inclusions (commonly known as Lewy bodies).

In Parkinson's disease, $\alpha$-synuclein oligomers in cerebrospinal fluid levels and the ratio of oligomeric to total $\alpha$-synuclein generally increase. This last increased ratio of oligomeric to total $\alpha$-synuclein has been associated with motor decline. Homovanillic acid and 3,4-dihydroxyphenylacetic acid are two primary metabolites of dopamine; they can be used as a cerebrospinal fluid markers of dopamine metabolism.

Several therapies for neurodegeneratives diseases are still on research [4]. An example is the new repurposing indication of Tasigna (nilotinib) by Novartis (Basel, Switzerland) for Parkinson's disease [5]. Nilotinib was first approved by the US FDA for adults with chronic myeloid leukemia. The range of doses in the treatment is of 600-800 mg.

Nilotinib is a broad-based tyrosine kinase inhibitor. Nilotinib has been shown high affinity to inhibit Abelson (c$\mathrm{Abl}$ ) and discoidin domain receptors (DDR1/2). In this study, researchers have shown that the drug could improve Parkinson's biomarkers at a lower dose. Researchers showed that nilotinib enters the brain in a dose-independent manner. In fact, $200 \mathrm{mg}$ of nilotinib increases the level of 3,4-dihydroxyphenylacetic acid and homovanillic acid. Nilotinib reduces plasma total $\alpha$-synuclein. Additionally, during this study, it was observed a significant increase of the triggering receptors on myeloid cells (TREM)-2, also suggesting an anti-inflammatory effect.

\section{Designing encapsulation process for new drugs}

Using Scheutjens-Fleer self-consistent field computations, researchers from Eindhoven University of Technology (Eindhoven, Netherlands) and Utrecht University (Utrecht, Netherlands) have discovered the parameters that govern the encapsulation of drugs [6]. They suggested that solubilization was regulated by enthalpic and entropic contributions. The spatial distribution of the drugs dramatically affects its release rate; some drugs might concentrate at the center of the micelle and slowly release whereas other drugs gather at the core-shell interface of the micelle and typically release fast. Therefore, controlling the location of the drugs in the micelle, it is possible to control the release rate of the drugs [7], select the best excipients [8] and reducing side effects associated with therapy and create personalized therapeutic treatments. The aim is to adjust the release of the drug to the individual needs of the patient.

\section{In vitro method to develop medicines for children \& older people}

The size and shape of oral solid dosage forms can significantly affect patient compliance. Multiparticulates are generally better tolerated by patients than conventional tablets. Multiparticulates can be designed in a range of particle size, offering dose flexibility $[9,10]$. Their acceptability potentially depends on rheology of the liquid carrier and palatability. The availability of predictive models to early characterize the formulation is the key to produce medicines that are easier to be taken by patients with different swallowing capabilities.

In this context, one example is the recent published in vitro model of oral cavity to access the swallowing process of the liquids dosage forms [11]. In this study, authors considered water and hydrocolloids as suspending vehicles. As model, the suspended phase consisted of cellulose-based pellets. Authors found that water-thin liquids were not optimal for a complete oral clearance of the solids. A minimum critical viscosity is required to enable a smooth swallow but high viscosity might negatively affect the swallowing. Finally, the sensory study did not show differences between the levels of thickness of the hydrocolloids. 


\section{New algae for critical limb ischemia}

Critical limb ischemia is a clinical syndrome of ischemic pain at rest or tissue loss related to peripheral artery disease. Up to $50 \%$ of people with critical limb ischemia will either die or need amputation within 1 year if blood flow is not well restored.

Currently, the blocked section of the artery must be either bypassed during surgery or widened using a stent. However, those methods will eventually fail in one out of three patients or are not possible to begin with. Ultimately, amputation is the only option.

On 20 March 2019, a new algae-based treatment based on 300- $\mu \mathrm{m}$ alginate capsules was published [12]. These alginate capsules encapsulated proangio/arteriogenic macrophages. After an intramuscular delivery into the murine ischemic hindlimb, the cell retention improved. This last fact led to an enhanced angiogenesis and arteriogenesis, and an improvement in limb perfusion.

The researchers will try to translate this research into human clinical trials.

\section{Repurposing drugs to breast cancer}

A new approach to identifying effective treatments for patients with advanced metastatic cancers was done by researchers from The Nagourney Cancer Institute (CA, USA) and The Albert Einstein Israelite Hospital (São Paulo, Brazil) [13]. A drug commonly used in kidney and liver cancers was applied for breast cancer. Sorafenib is a tyrosine kinase inhibitor and induces ferroptosis. Ferroptosis is a form of non-apoptotic programmed cell death and this iron-mediated process of cancer cell death is activated by sorafenib.

The reported case was a 56-year-old patient with palpable axillary adenopathy. Laboratory analyses confirmed the presence of an adenocarcinoma with widespread metastatic disease to bone and liver. In this case, the initial chemotherapy was ineffective. Researchers described the diagnostic evaluation, treatment and achievement of durable remission using a novel sorafenib-based drug combination, making more rational the drug choices in cancer treatment based upon each individual's unique features.

\section{Heart muscle regeneration}

Regeneration of human heart muscle after injury is extremely limited. Researchers at Duke-NUS Medical School (Singapore, Singapore) have developed an approach to regenerate heart muscle using stem cells [12]. They showed that laminin-221 promotes differentiation of pluripotent human embryonic stem cells toward cardiomyocyte lineage and downregulates pluripotency and teratoma-associated genes. In this case, they produced cardiovascular precursor cells from stem cells. These stem cell-derived cardiovascular progenitors were transplanted into myocardial infarction mice and differentiated into cardiac muscle fiber bundles. Those survived in the heart for at least 12 weeks and the heart functions also improved.

However, the previous approach has not yet met regulations set forth by the FDA and the EMA for regenerative therapies.

Both regulatory authorities require that stem-cell-derived precursors are prepared from human-only cells and in cultures that use clearly defined chemicals. It cannot exist animal components. Further research is now expected.

\section{Syndecan 1 in pancreatic cancer}

With an extremely poor prognosis, pancreatic cancer remains one of the most lethal cancers worldwide [14]. Pancreatic ductal adenocarcinoma remains recalcitrant to all forms of cancer treatment. Inhibition of oncogenic $K R A S$ and its signaling surrogates has yielded encouraging preclinical results with new therapeutic agents. A functional target-discovery platform to query KRAS-dependent changes of the pancreatic ductal adenocarcinoma surface was developed. This platform reveals that Syndecan 1 (SDC1, also known as CD138) is an upregulated protein at the cell surface by $K R A S$ [15]. Localization of SDC1 at the cell surface is essential for disease maintenance and progression.

The full work was published in Nature on 27 March 2019 [15].

\section{Targeting metastasis in cancer}

On 29 March 2019, a new experimental drug combination was tested for cancer therapy [16]. Researchers combined two drugs together to inhibit some cell processes like kinases ATR and Wee1, that is, Wee1 regulates cell-cycle progression whereas ATR is an apical kinase in the DNA-damage response. Using an orthotopic breast cancer xenograft mouse model, they showed a complete remission in six of nine cases, an inhibition tumor spread and 
prolonged survival. The toxicity studies showed that this combination was associated with minimal adverse side effects. Mechanistic studies showed that combined ATR and Wee1 inhibition acts during S and G2/M phases. ATR and Wee1 inhibitors are already in Phase I/II clinical trials.

\section{Brain tumor: a new combination}

In the first-line setting, high-grade gliomas are usually treated with a combination of surgery and chemoradiotherapy but recurrence is very common. Thus, new options are urgently required.

Arginine is an essential amino acid involved in cell signaling, proliferation, vasodilatation and hormone synthesis. Furthermore, arginine displays a key role in regulating the immune system. In general, normal cells express the argininosuccinate lyase and urea cycle enzymes argininosuccinate synthetase 1 (ASS1). Here, citrulline is converted to arginine thereby providing an endogenous supply. But in many tumors, ASS1 is downregulated, commonly through hypermethylation of the ASS1 promoter. This leads to increased pyrimidine synthesis and consequent tumor proliferation but renders the tumor dependent on exogenous arginine. This can be therapeutically exploited using pegylated arginine deiminase (ADI-PEG20 or pegargiminase) by depleting arginine in the blood.

A clinical trial (Phase I) set out to assess the safety of this new combination of ADI-PEG20, pemetrexed and cisplatin [17]. In this trial, plasma arginine was significantly suppressed below the baseline with a reciprocal increase in citrulline during the sampling period. The median progression-free survival was 5.2 months and the overall survival was 6.3 months. The treatment was well tolerated.

Further, Phase II trial to include a larger patient cohort to further assess efficacy is currently being developed.

\section{New approvals}

Roche

Lung cancer is by far the leading cause of cancer death, estimated to be responsible for nearly one in five cancer deaths [18]. Approximately 1.8 million people die as a result of lung cancer per year.

On 20 March 2019, Roche (Basel, Switzerland) announced that the FDA has accepted the use of Tecentriq ${ }^{\circledR}$ (atezolizumab) in combination with carboplatin and etoposide. This triple therapy was approved for the first-line treatment of adults with extensive-stage small-cell lung cancer. Atezolizumab is a monoclonal antibody planned to bind to PD-L1 expressed on tumor cells and tumor-infiltrating immune cells. By inhibiting PD-L1, Tecentriq ${ }^{\circledR}$ may enable the activation of $\mathrm{T}$ cells.

The Phase III IMpower133 study enrolled 403 people and showed that this combination helped people live significantly longer compared with carboplatin and etoposide. The safety of atezolizumab alone or in combination with carboplatin and etoposide was consistent with atezolizumab's safety profile previously described in literature.

\section{AstraZeneca}

Diabetes is a chronic disease that occurs either when the pancreas does not produce enough insulin or when the body cannot effectively use the insulin it produces [19,20]. In 2016, diabetes was the direct cause of 1.6-million deaths.

Thus, AstraZeneca (Cambridge, England) is investing in a portfolio of medicines for metabolic diseases like diabetes. On 27 March 2019, Forxiga (Dapagliflozin) was approved by the Japanese Ministry of Health, Labour and Welfare (Chiyoda, Tokyo, Japan) as an oral adjunct treatment to insulin for adults with Type 1 diabetes, whose glucose levels are not adequately controlled with insulin alone (HbA1c 7.5-10.5\%). Forxiga is a first-in-class oral selective inhibitor of human sodium-glucose co-transporter 2 (SGLT2). This medicine has been also shown additional benefits of weight loss and blood pressure control. Clinical trials with Forxiga demonstrated a clinically meaningful reduction of HbA1c levels after 24 weeks. The same happened with weight and total daily insulin dose [21]. In Europe, Forxiga was approved on 20 March 2019 as an adjunct treatment in adults' diabetic Type 1. This medicine is under regulatory review in the USA for the same indication. A decision by regulatory agency is expected in the second half of 2019.

\section{Boehringer Ingelheim}

Boehringer Ingelheim (Ingelheim am Rhein, Germany) has filled nintedanib in patients with systemic sclerosis for regulatory approval. Systemic sclerosis is also known as scleroderma-associated interstitial lung disease (SSc-ILD). Scleroderma is an uncommon disease characterized by thickening and scarring of connective tissue. The disease typically occurs at a young age - between 25 and 55 years. On the other hand, pulmonary fibrosis is a key driver 
of mortality in systemic sclerosis. Currently, there are no approved treatments for SSc-ILD. Boehringer Ingelheim aims to bring an approved treatment. The results relating to nintedanib in SSc-ILD will be shared with the scientific community during the American Thoracic Society Conference 2019 (17-22 May 2019, TX, USA).

\section{Novartis}

On 27 March 2019, Novartis announced that the FDA had approved Mayzent ${ }^{\circledR}$ (Siponimod). This medicine was approved for the treatment of adults with relapsing forms of multiple sclerosis. This approval was based on groundbreaking data from the Phase III trial, EXPAND study. Phase III EXPAND study was a randomized, double-blind and placebo-controlled study. It was done at 292 hospital clinics and specialized multiple sclerosis centers in 31 countries, and it compared the efficacy and safety of Mayzent ${ }^{\circledR}$ versus placebo [21].

Multiple sclerosis is a chronic disorder of the CNS that affects around 2.3 million people worldwide and there are three main forms: relapsing-remitting multiple sclerosis (RRMS), the most common form of the condition at diagnosis, secondary progressive multiple sclerosis (SPMS) and primary progressive multiple sclerosis (PPMS). More than $50 \%$ of patients with RRMS changed to SPMS within 15-20 years. This leads to a progressive accumulation of neurological disability.

Siponimod is a selective sphingosine 1-phosphate receptor modulator. It is indicated for the treatment of relapsing forms of multiple sclerosis, which selectively binds to S1P1 and S1P5 receptors. In relation to the S1P1 receptor, Siponimod prevents the lymphocytes from egressing the lymph nodes and, as a consequence, from entering the CNS of patients with multiple sclerosis. This leads to the anti-inflammatory effects. Additionally, Siponimod enters the CNS and directly binds to the S1P5 and S1P1 sub-receptors on specific cells in the CNS (oligodendrocytes and astrocytes) to promote re-myelination and prevent inflammation.

\section{Clarus Therapeutics}

On 27 March 2019, the FDA granted approval of Jatenzo ${ }^{\circledR}$ to Clarus Therapeutics (IL, USA). Jatenzo ${ }^{\circledR}$ is a testosterone undecanoate capsule for testosterone replacement therapy. The target population is adult men with primary hypogonadism (congenital or acquired) and hypogonadotropic hypogonadism (congenital or acquired). Hypogonadism is a condition in men in which the body does not produce enough testosterone.

FDA approval was based on a multicenter, randomized and open-label Phase II trial (TUne clinical trial). A total of 222 patients were randomized and dosed with Jatenzo ${ }^{\circledR}(n=166)$ and comparator $(n=56 ; 3: 1$ ratio), and this study showed $87 \%$ of hypogonadal men treated with Jatenzo ${ }^{\circledR}$ achieved a daily average testosterone level in the normal range. The efficacy criteria were satisfied, and the study met its primary end point. The safety profile was coherent with data generated in two previous Phase III trials. Jatenzo ${ }^{\circledR}$ was associated with an increase in average systolic blood pressure consistent with a currently marketed form of injectable testosterone but further refined the dose adjustment scheme.

\section{Examples of patents}

Severe valvular heart disease is responsible for approximately 28,000 deaths per year. In valvular heart diseases, aortic valve disease has a mortality rate of about $65 \%$. The treatment require surgical replacement by mechanical or tissue bioprosthetic valves. These valves are based in collagen and they have early degeneration. Therefore, a robust alternative is needed.

Accordingly, Wake Forest University Health Sciences (NC, USA) was granted with the US patent number $10,219,895$ on 5 March 2019 [22]. This patent describes a new nanofiber-based graft for heart valve replacement made by collagen protein blended with fibroin and a hemocompatible synthetic elastomeric polymer or elastomer, which is more robust.

US patent number 10,231,956 (Assignee Samumed, LLC), dated 19 March 2019 [23], provides 4-azaindazole compounds to potentially target several diseases such as cancer, angiogenesis, abnormal cellular proliferation, bone or cartilage disease, fibrotic disorders and osteoarthritis. These compounds are involved in the modulation of cellular events mediated by Wnt pathway signaling. The mechanism of action might have involved mutations or dysregulation of the Wnt pathway and/or of one or more of Wnt signaling components [11].

The Dana-Farber Cancer Institute, Inc. (MA, USA) and Alnylam Pharmaceuticals, Inc. (MA, USA) were granted with US patent number 10,233,452 (19 March 2019) [24]. The invention relates to dsRNA compositions targeting one or more EGLN genes, EGLN1, EGLN2 and/or EGLN3, and methods to inhibit these genes. This patent also discloses some claims about increasing erythropoietin production in diseases like anemia, hypoxia, neurological 
conditions including degeneration, renal disease or failure, and in some cancers including those of the blood, bone and marrow. It has surprisingly been discovered that synergistic effects are seen upon the administration of a mix or plurality of iRNA agents collectively targeting all three EGLN genes.

Alexion Pharmaceuticals, Inc. has been granted with the US patent number 10,239,937 on 26 March 2019 [25]. This patent describes new methods and materials for treatment of subjects suffering disorders that involve abnormal lysis of red blood cells like paroxysmal nocturnal hemoglobinuria, hemolytic anemias and other disorders that involve complement-mediated hemolysis, including intravascular and extravascular hemolysis [11].

The US granted patent number 10,239,919 to Avelas Biosciences, Inc. (CA, USA) on 26 March 2019 based on selective delivery molecules as therapeutic agents and/or imaging agents [26]. Those systems comprise different parts: a molecular transport or retention sequence (portion B); at least one cargo moiety (portion D) bound to portion A, B or X; X a linker; and a macromolecular carrier; and an acidic sequence (portion A). The cleavage of $\mathrm{X}$ linker will allow the uptake or retention of portion B and the attached cargo into cells and tissue.

On 26 March 2019 Alnylam Pharmaceuticals, Inc. was awarded with US patent number 10,238,749, providing iRNA compositions for treating disorders that would benefit from inhibiting or reducing the expression of a specific gene [27]. As example, these diseases can be angiotensinogen-associated diseases such as hypertension.

\section{Other news}

According to more recent data, Europe accounts for $20 \%$ of global R\&D investment. Europe is responsible for a third of all high-quality scientific publications [28]. On 20 March 2019, EU Commission announced the new provisional agreement on Horizon Europe. This program will continue to drive scientific excellence through, for example, the European Research Council and Marie Skłodowska-Curie fellowships. The preliminary political agreement is now subject to formal approval by the European Parliament and Council during next May.

On 28 March 2019, The Committee for Medicinal Products for Human Use (CHMP) from EMA's adopted a positive opinion for Zynteglo ${ }^{\circledR}$ [29]. Zynteglo ${ }^{\circledR}$ is indicated for the treatment of transfusion-dependent $\beta$ thalassaemia, a rare disease that causes severe anemia. It was first designated on January 2013 as an orphan medicinal product. Zynteglo ${ }^{\circledR}$ is an autologous $\mathrm{CD} 34^{+}$cell-enriched population and contains hematopoietic stem cells transduced with lentiviral vector encoding the $\beta^{\mathrm{A}-\mathrm{T} 87 \mathrm{Q}}$-globin gene. It is now intended for the management of disease for adults and children older than 12 years who have no matching donor for a stem cell transplant.

In addition, CHMP recommended extensions of indications for Revlimid, Mozobil and Imnovid [29].

Finally, after re-examination requested, CHMP has confirmed that omega-3 fatty acid medicines containing ethyl ester of eicosapentaenoic acid and docosahexaenoic acid are not effective in preventing further cardiovascular problems in patients who have had a heart attack [29]. No safety concerns were found. This re-examination does not affect the authorization of omega-3 fatty acid medicines for the treatment of hypertriglyceridemia.

Financial \& competing interest disclosure

This work was supported in part by UID/DTP/04138/2019 from National Funds, Fundação para a Ciência e para a Tecnologia, FCT, Portugal. The author has no other relevant affiliations or financial involvement with any organization or entity with a financial interest in or financial conflict with the subject matter or materials discussed in the manuscript apart from those disclosed.

No writing assistance was utilized in the production of this manuscript.

\section{References}

1. de Almeida BP, Vieira AF, Paredes J, Bettencourt-Dias M, Barbosa-Morais NL. Pan-cancer association of a centrosome amplification gene expression signature with genomic alterations and clinical outcome. PLOS Comput. Biol. 15(3), e1006832 (2019).

2. Chabanon RM, Muirhead G, Krastev DB et al. PARP inhibition enhances tumor cell-intrinsic immunity in ERCC1-deficient non-small cell lung cancer. J. Clin. Invest. 129(3), 1211-1228 (2019).

3. Ray KK, Bays HE, Catapano AL et al. Safety and efficacy of bempedoic acid to reduce LDL cholesterol. N. Engl. J. Med. 380(11), 1022-1032 (2019).

4. Faustino C, Rijo P, Reis CP. Nanotechnological strategies for nerve growth factor delivery: therapeutic implications in Alzheimer's disease. Pharmacol. Res. 120, 68-87 (2017).

5. Pagan FL, Hebron ML, Wilmarth B et al. Pharmacokinetics and pharmacodynamics of a single dose nilotinib in individuals with Parkinson's disease. Pharmacol. Res. Perspect. 7(2), e00470 (2019).

6. Ianiro A, González García A, Wijker S, Patterson JP, Esteves ACC, Tuinier R. Controlling the spatial distribution of solubilized compounds within copolymer micelles. Langmuir 35(13), 4776-4786 (2019). 
7. Faustino C, Serafim C, Rijo P, Reis CP. Bile acids and bile acid derivatives: use in drug delivery systems and as therapeutic agents. Expert Opin. Drug Deliv. 13(8), 1133-1148 (2016).

8. Abrantes CG, Duarte D, Reis CP. An overview of pharmaceutical excipients: safe or not safe? J. Pharm. Sci. 105(7), $2019-2026$ (2016).

9. Reis CP, Gomes A, Rijo P et al. Development and evaluation of a novel topical treatment for acne with azelaic acid-loaded nanoparticles. Microsc. Microanal. 19(05), 1141-1150 (2013).

10. Reis CP, Martinho N, Rosado C, Fernandes AS, Roberto A. Design of polymeric nanoparticles and its applications as drug delivery systems for acne treatment. Drug Dev. Ind. Pharm. 40(3), 409-417 (2014).

11. Marconati M, Lopez F, Tuleu C, Orlu M, Ramaioli M. In vitro and sensory tests to design easy-to-swallow multi-particulate formulations. Eur. J. Pharm. Sci. 132, 157-162 (2019).

12. Ludwinski FE, Patel AS, Damodaran G et al. Encapsulation of macrophages enhances their retention and angiogenic potential. NPJ Regen. Med. 20, 6 (2019).

13. Usón JuniorPLS, Wagner J, de Corpa MVN, Coelho IM, Nagourney RA, Yamaguchi NH. Metastatic carcinoma of unknown primary with complete metabolic response following sorafenib-based chemotherapy. SAGE Open Med. Case Reports 7, 1-4 (2019).

14. Rebelo A, Molpeceres J, Rijo P, Pinto Reis C. Pancreatic cancer therapy review: from classic therapeutic agents to modern nanotechnologies. Curr. Drug Metab. 18(4), 346-359 (2017).

15. Yao W, Rose JL, Wang W et al. Syndecan 1 is a critical mediator of macropinocytosis in pancreatic cancer. Nature 568(7752), 410-414 (2019).

16. Bukhari AB, Lewis CW, Pearce JJ, Luong D, Chan GK, Gamper AM. Inhibiting Wee1 and ATR kinases produces tumor-selective synthetic lethality and suppresses metastasis. J. Clin. Invest. 129(3), 1329-1344 (2019).

17. Hall PE, Lewis R, Syed N et al. A Phase I study of pegylated arginine deiminase (pegargiminase), cisplatin, and pemetrexed in argininosuccinate synthetase 1-deficient recurrent high-grade glioma. Clin. Cancer Res. 25(9), 2708-2716 (2019).

18. WHO: IARC. Cancer fact sheets: lung cancer (2019). http://gco.iarc.fr/today

19. Reis CP, Figueiredo IV, Carvalho RA et al. Toxicological assessment of orally delivered nanoparticulate insulin. Nanotoxicology 2(4), 205-217 (2008).

20. Reis CP, Damgé C. Nanotechnology as a promising strategy for alternative routes of insulin delivery. Methods Enzymol. 508, 271-294 (2012).

21. Dandona P, Mathieu C, Phillip M et al. Efficacy and safety of dapagliflozin in patients with inadequately controlled Type 1 diabetes (DEPICT-1): 24 week results from a multicentre, double-blind, Phase III, randomised controlled trial. Lancet Diabetes Endocrinol. 5(11), 864-876 (2017).

22. Wagner W, Nicole L, Wang R, Argenta L, Morykwa M. Nanofiber-based graft for heart valve replacement and methods of using the same. US patent 10219895 (2019).

23. Kc SK, Wallace DM, Jianguo C, Chiruta C, Hood J. 3-(1H-pyrrolo[3,2-C]pyridin-2-YL)-1 H-pyrazolo[4,3-B]pyridines and therapeutic uses thereof. US patent 102319563 (2019).

24. Kaelin Jr, William G, Kotelianski V, Querbes W, Bettencourt B. Compositions and methods for increasing erythropoietin (EPO) production. US patent 10233452 (2019).

25. Holers VM, Risitano AM. Treatment of paroxysmal nocturnal hemoglobinuria, hemolytic anemias and disease states involving intravascular and extravascular hemolysis. US patent 10239937 (2019).

26. Gonzalez J, Liu J. Selective delivery molecules and methods of use. US patent 10239919 (2019).

27. Donald F, Brian B, Klaus C et al. Angiotensinogen (AGT) iRNA compositions and methods of use thereof. US patent 10238749 (2019).

28. European Commission - press releases - press release - EU budget for 2021-2027: commission welcomes provisional agreement on Horizon Europe, the future EU research and innovation programme. (2019). http://europa.eu/rapid/press-release_IP-19-1676_en.htm

29. EMA. Meeting highlights from the Committee for Medicinal Products for Human Use (CHMP) 25-28 March 2019 (2019). https://www.ema.europa.eu/en/news/meeting-highlights-committee-medicinal-products-human-use-chmp-25-28-march-2019 
